\title{
Glide symmetry protected higher-order topological insulators from semimetals with butterfly-like nodal lines
}

\author{
Xiaoting Zhou $\mathbb{D}^{1 凶}$, Chuang-Han Hsu $\mathbb{D}^{2,3}$, Cheng-Yi Huang ${ }^{1,3}$, Mikel Iraola $\mathbb{D}^{4,5}$, Juan L. Mañes ${ }^{5}$, Maia G. Vergniory ${ }^{4,6}$, Hsin Lin $\mathbb{D}^{3}$ and
} Nicholas Kioussis (iD ${ }^{1 凶}$

Most topological insulators (TIs) discovered today in spinful systems can be transformed from topological semimetals (TSMs) with vanishing bulk gap via introducing the spin-orbit coupling (SOC), which manifests the intrinsic links between the gapped topological insulator phases and the gapless TSMs. Recently, we have discovered a family of TSMs in time-reversal invariant spinless systems, which host butterfly-like nodal-lines (NLs) consisting of a pair of identical concentric intersecting coplanar ellipses (CICE). In this Communication, we unveil the intrinsic link between this exotic class of nodal-line semimetals (NLSMs) and a $\mathbb{Z}_{4}=2$ topological crystalline insulator (TCl), by including substantial SOC. We demonstrate that in three space groups (i.e., Pbam (No.55), $\mathrm{P} 4 / \mathrm{mbm}$ (No.127), and $\mathrm{P}_{2} / \mathrm{mbc}$ (No.135)), the TCl supports a fourfold Dirac fermion on the (001) surface protected by two glide symmetries, which originates from the intertwined drumhead surface states of the CICE NLs. The higher order topology is further demonstrated by the emergence of one-dimensional helical hinge states, indicating the discovery of a higher order topological insulator protected by a glide symmetry.

npj Computational Materials (2021) 7:202; https://doi.org/10.1038/s41524-021-00672-9

\section{INTRODUCTION}

The discovery of the quantum spin Hall effect $(\mathrm{QSHE})^{1-3}$ and topological insulators (TIs) ${ }^{4-6}$ which are protected by time-reversal symmetry (TRS), has indicated that symmetry plays a crucial role in classifying the topology of free fermion states ${ }^{7-9}$. Subsequently, the concept has been generalized to spatial symmetries in crystalline systems. For instance, topological crystalline insulators $(\mathrm{TCls})^{10}$ are protected by other space-group symmetries $(\mathcal{Q})^{11,12}$, such as mirror ${ }^{13}$ and rotational symmetries ${ }^{14-16}$. Consequently, such type of systems are known to harbor symmetry-protected topological (SPT) phase ${ }^{17}$.

In free fermion systems, SPT insulators harbor a central paradigm referred to as the bulk-boundary correspondence ${ }^{18}$. A $d$-dimensional bulk with gapped excitations hosts anomalous gapless, topologically nontrivial boundary states in lower $(d-1)$ dimensions $s^{4,19}$. Recently, a higher-order bulk-boundary correspondence has been uncovered in types of $\mathrm{TCl}$, which exhibit a gapped $(d-1)$-dimensional boundary but a gapless $(d-2)$-dimensional boundary ${ }^{14,20-22}$. Hence, this type of TCls are dubbed higher-order topological insulators (HOTIs) ${ }^{14,20-27}$. In general, an $n$th order topological insulator describes a $\mathrm{TCl}$ in $d$-dimensions having symmetry protected $(d-n)$-dimensional gapless boundary states, but gapped otherwise, when the sample geometry is properly selected, being compatible with $\mathcal{Q}$. HOTIs protected by various symmetries have been studied, such as $\mathcal{C}_{4}^{z} \mathcal{T}$, the mirror symmetry ${ }^{22}$, and the inversion symmetry ${ }^{23}$, respectively.

In contrast to the gapped topological phase, a topological semimetal (TSM) has gapless bulk band structures, which are characterized by the topologically robust band-crossings manifolds between occupied and unoccupied bands in momentum space. Among them, nodal-line semimetals (NLSMs) ${ }^{28,29}$, which harbor one-dimensional (1D) nodal lines (NLs), possess the highest variability. NLSMs with NLs integrated in various configurations have been studied under the assumption that the spin-orbit coupling (SOC) is negligible or absent, e.g., a chain link ${ }^{30-32}$, a Hopf link $^{31}$, and a knot $^{33}$.

However, from another perspective, it is intuitive to raise the question whether additional topology could be unearthed when these intricate degenerate links are gapped out by substantial SOC. The intrinsic link between gapped TIs or TCls and gapless TSMs is essential to trace the origin of the topology of the insulating phase due to the band inversions and the evolution of the surface states. It has been known that a $\mathbb{Z}_{2}$ strong $\mathrm{Tl}$ can be realized from NLSMs with a single nodal ring when SOC is included $^{34-36}$, and a HOTI from NLSMs with monopole nodal lines ${ }^{24}$, which represent the intrinsic link between a gapped topological phase and a gapless TSM. Nevertheless, similar studies for NLSMs with complex NL configurations remain deficient. In this work, we unveil the intrinsic link between an exotic class of nodalline semimetals (NLSMs) and a $\mathbb{Z}_{4}=2$ topological crystalline insulator (TCl). This type of NLSM has been discovered and reported in ref. ${ }^{37}$ recently in spinless systems, which hosts a butterfly-like NL consisting of a pair of concentric intersecting coplanar ellipses (CICE) residing on a plane in $k$ space as shown in Fig. 1a.

In this paper, we include the Pbam (No.55) symmetry-invariant SOC in a minimal tight-binding (TB) model which exhibits CICE in ref. ${ }^{37}$, and (i) demonstrate that the CICE act as the origin of a $\mathrm{TCl}$ protected by two glide symmetries. With substantial SOC, as shown in Fig. $1 \mathrm{~b}$, the CICE become anticrossing, thus driving a phase transition from NLSM to a TCl with $\mathbb{Z}_{4}=2^{38-41}$ due to the fact that the CICE are essentially sets of NLs stemming from the double-band-inversion (DBI). (ii) Consequently, the intertwined drumhead surface states (DSSs) on the (001) surface (wallpaper

\footnotetext{
${ }^{1}$ Department of Physics and Astronomy, California State University, Northridge, CA 91330, USA. ${ }^{2}$ Department of Electrical and Computer Engineering, Faculty of Engineering, National University of Singapore, Singapore 117583, Singapore. ${ }^{3}$ Institute of Physics, Academia Sinica, Taipei 115201, Taiwan. ${ }^{4}$ Donostia International Physics Center, DonostiaSan Sebastian 20018, Spain. ${ }^{5}$ Department of Condensed Matter Physics, University of the Basque Country UPV/EHU, Apartado 644, Bilbao 48080, Spain. ${ }^{6}$ IKERBASQUE, Basque Foundation for Science, Maria Diaz de Haro 3, Bilbao 48013, Spain. ${ }^{\bowtie}$ email: physxtzhou@gmail.com; nick.kioussis@csun.edu
} 

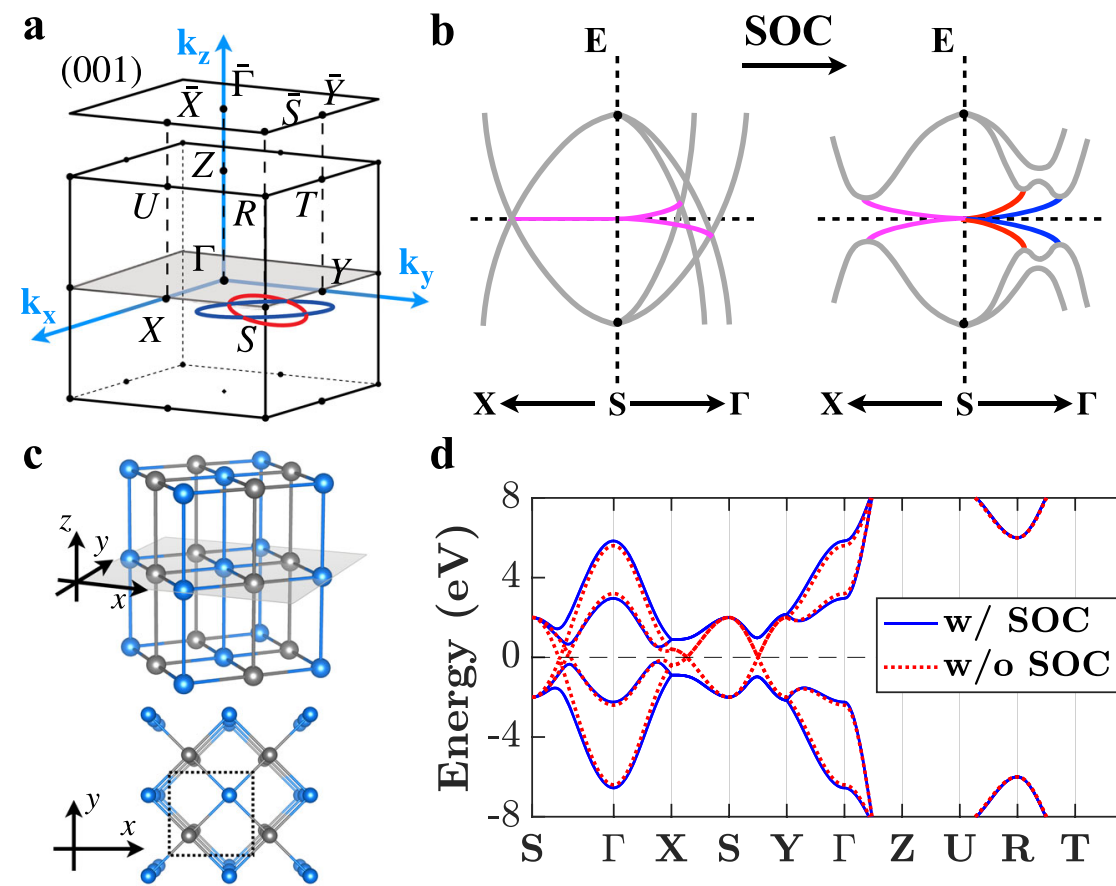

d

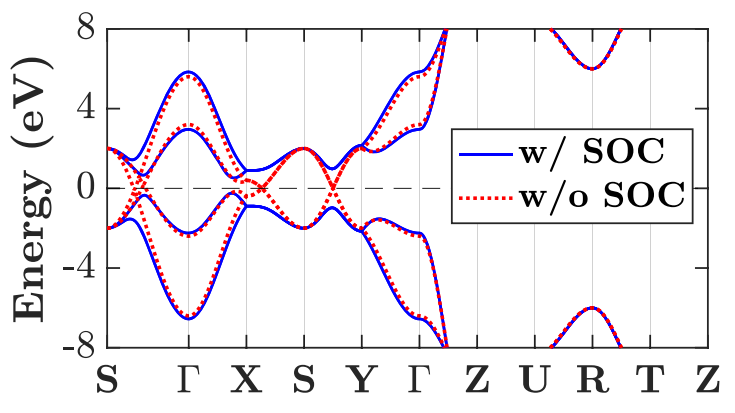

Fig. 1 Phase transition from a NLSM to a TCl, crystal structure, and band structures. a Brillouin zone (BZ) of bulk and the projected (001) surface in SG Pbam (No. 55), with the high-symmetry points. The CICE nodal lines are on the $x y$-plane centered at $S$ point. $\mathbf{b}$ Schematic band structures demonstrating the SOC-driven transition from a NLSM exhibiting the CICE NLs ${ }^{37}$ (left panel) to a TCI (right panel). Gray denotes the bulk states, and the magenta, red and blue indicate the (001) surface states, i.e., the interwined DSSs of the NLSM (left panel), and the TSSs of the TCl with two intertwined Dirac cones (right panel). c Orthorhombic crystal structure of the lattice model in Eqs. (1) and (2), consisting of a lattice with two sublattices A (in blue) and B (in gray). d Band structure of the model with and without SOC, respectively.

group (WG) pgg) stemming from the CICE nodal lines, evolve to the topological surface states (TSSs) with a fourfold Dirac fermion ${ }^{42,43}$ protected by the two glide symmetries, corresponding to the $\mathrm{TCl}$ as shown in Figs. $1 \mathrm{~b}$ and $2 \mathrm{~d}$, respectively. (iii) We further uncover the higher-order topology of the system featuring the 1D helical hinge states when the sample geometries are distinctively and properly selected, which indicates the discovery of the three-dimensional (3D) HOTI protected by a nonsymmorphic glide symmetry.

\section{RESULTS}

\section{The lattice model}

The CICE can be sustained by two glide mirror symmetries and only nine space groups (SGs) are feasible to host $\mathrm{it}^{37}$. The minimal 4-band TB model for the spinless CICE NLSM in SG Pbam (No. 55) is a lattice, consisting of two sublattices denoted by $A$ (gray) and $B$ (blue) which occupy the 2 a Wyckoff position at $\mathbf{r}_{A}=$ $(0,0,0)$ and $\mathbf{r}_{B}=\left(\frac{1}{2}, \frac{1}{2}, 0\right)$ in the unit cell (see Fig. 1c for the structure). There are two orbitals, $p_{z}$ and $d_{x y}$, for each sublattice, described by the Pauli matrix $\boldsymbol{\sigma}$ and $\boldsymbol{\tau}$ for the $A$ and $B$ sublattices, respectively. $\sigma_{0}$ and $\tau_{0}$ are identity matrices. The SG Pbam (No.55) can be generated by the mirror symmetry, $\mathcal{M}_{z}=\left\{m_{001} \mid 000\right\}$, and the two glide-mirror symmetries, $\mathcal{G}_{x}=\left\{m_{100} \mid \frac{1}{2} \frac{1}{2} 0\right\}$ and $\mathcal{G}_{y}=\left\{m_{010} \mid \frac{1}{2} \frac{1}{2} 0\right\}$, normal to the [100] and [010] directions, respectively, accompanied by a translation of $\left[\frac{1}{2} \frac{1}{2} 0\right]$. For a spinless system, employing the basis $\Psi=\left(p_{z}^{A}, d_{x y}^{A}, p_{z}^{B}, d_{x y}^{B}\right)^{T}$, the symmetry-constrained TB Hamiltonian is of the form,

$$
\begin{aligned}
H_{0}(\mathbf{k})= & {\left[\left(a \cos k_{x}+\beta \cos k_{y}+\gamma \cos k_{z}\right)+\delta_{0}\right] \tau_{0} \sigma_{3} } \\
& +\cos \frac{k_{x}}{2} \cos \frac{k_{y}}{2} \cos k_{z}\left(\lambda_{10} \tau_{1} \sigma_{0}+\lambda_{13} \tau_{1} \sigma_{3}\right) \\
& +\sin k_{z}\left(\lambda_{32} \tau_{3} \sigma_{2}\right)+\sin \frac{k_{x}}{2} \sin \frac{k_{y}}{2} \sin k_{z}\left(\lambda_{12} \tau_{1} \sigma_{2}\right),
\end{aligned}
$$

where $a, \beta, \gamma$, and $\lambda_{i j}$ are hopping strength, and $\delta_{0}$ represents the chemical potential. As discussed in detail in ref. ${ }^{37}$, the CICE emerge on the mirror plane (gray shaded area in Fig. 1a) centered at the high symmetry $k$ point $S=(\pi, \pi, 0)[R=(\pi, \pi, \pi)]$, under the conditions $\left\{a \delta_{S(R)}<0 \cap a \beta>0 \cap a \neq \beta\right\}$, where $\delta_{S, R}=\delta_{0}-(a+\beta \mp \gamma)$. The various terms in Eq. (1) describe the pair of concentric elliptic NLs, the NL anisotropy, and the angle between the NLs (see details in ref. ${ }^{37}$ ). Since the CICE are composed of two NLs, it is anticipated to observe a pair of DSS ${ }^{28}$ intertwined on the (001) surface.

\section{Topological crystalline insulator}

We consider the effect of SOC, and the minimal TB model contains eight spinful bands. The minimal SOC Hamiltonian in SG Pbam (No. 55), $H_{\text {soc }}(\mathbf{k})$, to gap out the CICE-NL is of the form,

$$
\begin{aligned}
H_{\text {SOC }}(\mathbf{k})= & \sum_{i=1}^{3} \zeta_{01 i} \sin k_{i} \Gamma_{01 i} \\
& +\zeta_{233} \cos \frac{k_{x}}{2} \cos \frac{k_{y}}{2} \cos k_{z} \Gamma_{233}+\zeta_{111} \sin \frac{k_{x}}{2} \cos \frac{k_{y}}{2} \cos k_{z} \Gamma_{111} \\
& +\zeta_{112} \cos \frac{k_{x}}{2} \sin \frac{k_{y}}{2} \cos k_{z} \Gamma_{112}+\zeta_{223} \sin \frac{k_{x}}{2} \sin \frac{k_{y}}{2} \sin k_{z} \Gamma_{223},
\end{aligned}
$$

where $\zeta_{i j k}$ denotes SOC strength, and $\Gamma_{i j k}=\tau_{j} \sigma_{j} s_{k}(i, j, k \in\{0,1,2,3\})$. $s_{0,1,2,3}$ are identity matrix and Pauli matrices operating in spin space, respectively. The band structure of $H_{0}$ and $H=H_{0}+H_{S O C}$ are shown in Fig. 1d by the red and blue curves, respectively. In the presence of SOC, the CICE-NL TSM evolves into an insulating phase. The parameters are tuned to allow the system to host a single CICE centered at the $S$ point in the absence of SOC and to have no additional band inversions at other $\mathrm{k}$ points including SOC. In addition to $\left\{a \delta_{S}<0 \cap a \beta>0 \cap a \neq \beta\right\}$, either condition $\left\{\delta_{S}<0 \cap \delta_{R}>0 \cap \delta_{\Gamma, Z}>\sqrt{\lambda_{+}^{2}+\zeta_{233}^{2}}+\sqrt{\lambda_{-}^{2}+\zeta_{233}^{2}}\right\}$ or $\left\{\delta_{S}>0 \cap \delta_{R}<0 \cap \delta_{\Gamma, Z}<-\sqrt{\lambda_{+}^{2}+\zeta_{233}^{2}}-\sqrt{\lambda_{-}^{2}+\zeta_{233}^{2}}\right\}$, should be 


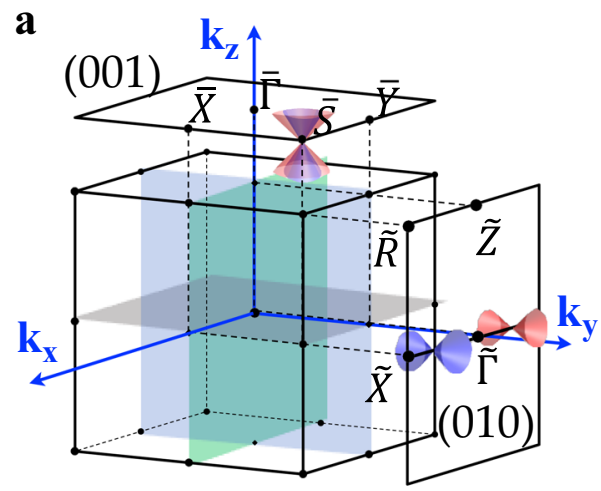

d

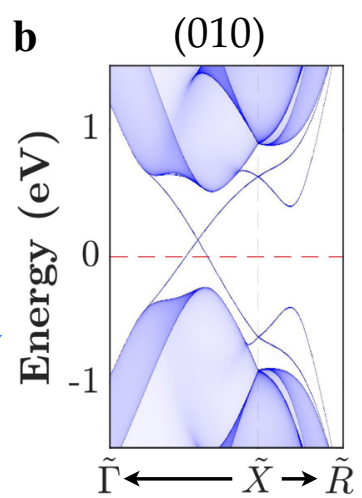

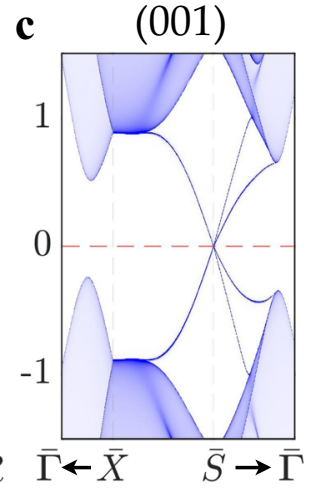

e

(001)
Intertwined DSSs

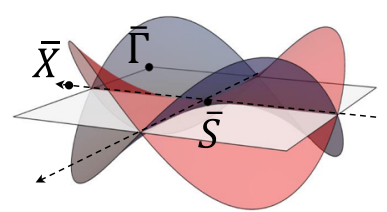

Fourfold Dirac TSSs

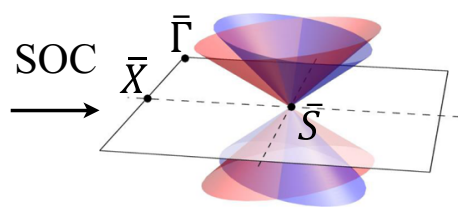

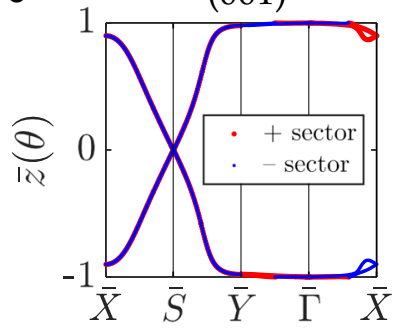

Fig. 2 Topological surface states and the evolution from DSSs to TSSs. a Brilliouin zones for the bulk, the projected (001) and (010) surfaces, on which the predicted positions of the fourfold Dirac fermion and Dirac cones are shown schematically. Surface band spectrum of the $\mathbf{b}$ (010) and $\mathbf{c}(001)$ surfaces along the high-symmetry k paths, where the fourfold Dirac fermion emerges at $\bar{S}$ on the (001) surface and a Dirac cone lies along the $\tilde{\Gamma}-\tilde{X}$ direction of the (010) surface, respectively. $\mathbf{d}$ Schematic of the SOC-driven transition of the (001) surface states from the intertwined DSSs stemming from the CICE NLSM ${ }^{37}$ (left panel) to the fourfold Dirac fermion at $\bar{S}$ of the TCI (right panel). e The $z$-directed Wilson loop along the high symmetry directions for occupied states hosting positive (+sector, red) and negative ( - sector, blue) surface glide eigenvalues, indicating a bulk topology of $\left(X_{x}, X_{y}\right)=(2,2)$.

satisfied, where $\lambda_{ \pm}=\lambda_{10} \pm \lambda_{13}$ and $\delta_{\Gamma, Z}=\delta_{0}+(\alpha+\beta \pm \gamma)$. If additional band inversions emerge beyond the one which gives rise to the $\mathrm{CICE}$, the band topology may be changed in the presence of SOC, and the semimetal may evolve to distinct insulating phases, although the CICE nodal lines may still exist in the absence of SOC.

In order to determine its band topology, we implement the symmetry-indicator theory ${ }^{38,40,41}$. Crystals in the SG Pbam (No. 55) are characterized by four symmetry indicators (SIs) ${ }^{38,40,41}$, three $\mathbb{Z}_{2}$ weak $\mathrm{Tl}$ indices and one $\mathbb{Z}_{4}$ index. The $\mathbb{Z}_{4}$ index is defined as, $\mathbb{Z}_{4} \equiv \frac{1}{4} \sum_{K \in \text { TRIMs }}\left(n_{K}^{+}-n_{K}^{-}\right) \bmod 4$, where $n_{K}^{+}\left(n_{K}^{-}\right)$is the number of occupied bands with parity $+(-)$ at the TRIM points $K$. Due to the nonsymmorphic symmetries, bands are four-fold degenerate at all TRIM points except $\Gamma$. Besides, since inversion $\mathcal{I}$ anticommutes with the glide, $\mathcal{G}$, or screw, $\mathcal{S}$, symmetry operations (here $\mathcal{S}$ includes $\mathcal{S}_{y}=2_{1}^{[010]}=\left\{2_{010} \mid \frac{1}{2} \frac{1}{2} 0\right\}$, and $\left.\mathcal{S}_{x}=2_{1}^{[100]}=\left\{2_{100} \mid \frac{1}{2} \frac{1}{2} 0\right\}\right)$, at $X, U, Y$, and $T$, the parity of each four-fold degenerate state must be $(+,+,-,-)$, which does not contribute to $\mathbb{Z}_{4}$. By enumerating the parity of the states at other TRIM points, we obtain $\mathbb{Z}_{2,2,2,4}=(0,0,0,2)$, corresponding to eight possible topological states $^{41}$. To further narrow down the possible phases, we have calculated the mirror Chern numbers of $\mathcal{M}_{z}$ and $C_{m_{0, \pi}^{001}}\left(m_{0, \pi}^{001}\right.$ denotes the $k_{z}=0, \pi$ mirror planes), following the method implemented in ref. ${ }^{16}$. We find that $C_{m_{0, \pi}^{001}}=(2,0)$. The corresponding Dirac surface states on the (010) surface are shown in Fig. $2 b$, where the relevant $k$ points and the schematic locations of the Dirac cones are illustrated in Fig. 2a. Therefore, given that $C_{m_{0, \pi}^{01}}=(2,0)$, there are two possible topological phases, which are listed in Table 1. The first one is $\mathcal{S}$-protected $\mathrm{TCl}$ with $v_{2_{1}^{[010]}}=v_{2_{1}^{[100]}}=1$, while the second one is $\mathcal{G}$-protected $\mathrm{TCl}$ with nontrivial $\mathbb{Z}_{2}$ topological invariants $v_{g_{a}^{(010)}}$ and $v_{g_{b}^{(100)}}$ (where
Table 1. The two possible topological states with SIs $\mathbb{Z}_{2,2,2,4}=$ $(0,0,0,2)$ and mirror Chern number $C_{m^{(001)}}=(2,0)$ in SG Pbam (No. $55)^{41}$. The $\mathbb{Z}$ invariants, $C_{m_{(001)}}$, are the mirror Chern numbers for the mirror planes $\mathcal{M}_{(001)}$ with $k_{z}^{0, \pi}=0, \pi$, respectively. All the listed $v^{\prime}$ s are $\mathbb{Z}_{2}$ classified topological invariants. The set $\left(v_{0} ; v_{1} v_{2} v_{3}\right)$ are the invariants for 3D $\mathbb{Z}_{2}$ TIs. $v_{g_{a}^{010}}$ and $v_{g_{b}^{100}}$ represent the invariants for the glide symmetries $\mathcal{G}_{y}$ and $\mathcal{G}_{x}$, respectively. $v_{\mathcal{I}}$ is the inversion $\mathcal{I}$ protected TCl index, where $v_{\mathcal{I}}=1$, features the hinge states in a 3D finite geometry preserving $\mathcal{I}$. $v_{2^{001}}, v_{2_{1}^{010}}$ and $v_{2_{1}^{100}}$ denote the invariants for the rotational and screw symmetries $2_{[001]}, 2_{1}^{[010]}$ and $2_{1}^{[100]}$, respectively.

\begin{tabular}{llllllll}
\hline$\left(v_{0} ; v_{1} v_{2} v_{3}\right)$ & $C_{m_{0, \pi}^{(001)}}$ & $v_{g_{a}^{010}}$ & $v_{g_{b}^{100}}$ & $v_{\mathcal{I}}$ & $v_{2^{001}}$ & $v_{2_{1}^{010}}$ & $v_{2_{1}^{100}}$ \\
\hline$(0 ; 000)$ & $(2,0)$ & 0 & 0 & 1 & 0 & 1 & 1 \\
$(0 ; 000)$ & $(2,0)$ & 1 & 1 & 1 & 0 & 0 & 0 \\
\hline
\end{tabular}

$g_{a}^{(010)}=\mathcal{G}_{y}=\left\{m_{010} \mid \frac{1}{2} \frac{1}{2} 0\right\}$, and $\left.g_{b}^{(100)}=\mathcal{G}_{x}=\left\{m_{100} \mid \frac{1}{2} \frac{1}{2} 0\right\}\right)$. Note that the nontrivial characteristics of the bands agree with the analysis from the elementary band representations (EBRs) ${ }^{44-46}$. The physical EBRs for the 2a Wyckoff position ${ }^{47-49}$ require that the parity of $\Gamma, Z, S$, and $R$ has the same sign. However, because of the double band inversion at $\mathrm{S}$ guaranteed by the CICE-NL, both valence and conduction bands violate the physical EBRs, suggesting the emergence of nontrivial topology.

To further determine the topological phase certainly, we have also investigated the (001) surface bands, because the presence of topological surface states on the (001) surface excludes the scenario of $\mathcal{S}$-protected TCl. The (001) surface bands are shown in Fig. 2c, where the (001) surface BZ and the corresponding high symmetry $k$ points are displayed Fig. 2a. Interestingly, the calculations reveal the emergence of nontrivial surface states 
around $\bar{S}$ which are composed of two intertwined surface Dirac cones, shown schematically in Fig. 2d (right panel). We refer to these topological surface states (TSSs) as fourfold Dirac fermions, which are of a particular type of wallpaper fermions ${ }^{42}$. As Fig. $2 d$ shows, one can also obtain the fourfold Dirac fermions via SOCinduced splitting of the intertwined DSSs of the CICE TSM ${ }^{37}$, where the degenerate dispersions along $\bar{S}-\bar{X}(\bar{Y})$ are guaranteed by $\mathcal{G}$. Consequently, the CICE-NL induced topological phase belongs to the $\mathcal{G}$-protected $\mathrm{TCl}$. In the following, we provide more physical insights on this $\mathrm{TCl}$ phase and the fourfold Dirac fermions.

In general, for time reversal symmetric systems, strong topological insulators (STIs) with a single band inversion at one TRIM point can be regarded as an elementary building block of the nontrivial insulating phase $\mathrm{e}^{15,40}$. For each STI, the topological surface states of the (001) surface can be described by the Hamiltonian, $h_{\mathbf{k}}=k_{x} s_{2}-$ $k_{y} s_{1}{ }^{40}$. The gapless feature of $h_{\mathbf{k}}$ is protected by the TRS operator, $\mathcal{T}=-i s_{2} \mathcal{K}$, where $\mathcal{K}$ is the complex conjugation operator. For the current case, since there is a $\mathrm{DBI}$ at the $\bar{S}$ point, the induced $\mathrm{TCl}$ phase can be viewed as two copies of STIs. Accordingly, for the TSSs of the TCl, the only allowed TR invariant mass term takes the form, $M=m \mu_{2} \otimes s_{3}$, where $\mu_{1,2,3}$ are the Pauli matrices acting on the two copies of $h_{\mathbf{k}}$, and $m$ is constant. If $M$ can be prohibited by any spatial symmetry $\mathcal{Q}$, the anomalous gapless surface states will persist, indicating that the existing topology is protected by $\mathcal{Q}$, which can be $\mathcal{G}_{x}$ and $\mathcal{G}_{y}$, as derived below.

At $\bar{S}$, the eigenvalues of $\mathcal{G}_{x}$ and $\mathcal{G}_{y}$ are \pm 1 . To preserve TRS, the only available representations are $\mathcal{G}_{x}=\mu_{2} \otimes s_{1}$ and $\mathcal{G}_{y}=\mu_{2} \otimes s_{2}$, which in turn lead to the rotational symmetry about the $z$-axis $C_{2 z}\left(=\mathcal{G}_{x} \times \mathcal{G}_{y}\right)=-i \mu_{0} \otimes s_{3}$. Obviously, $M$ cannot survive with $\mathcal{G}_{x}$ and $\mathcal{G}_{y}$, but is allowed by $C_{2 z}$. Consequently, there exist representations for $\mathcal{G}_{x}$ and $\mathcal{G}_{y}$ to support the (001) TSSs at $\bar{S}$, which is the fourfold Dirac fermion shown in Fig. $2 d$ described by the $k \cdot p$ Hamiltonian

$$
\begin{aligned}
H_{T S S}(\mathbf{q})= & g_{0}\left(q_{x} s_{2}-q_{y} s_{1}\right)+\sum_{i=1,3} g_{i} \mu_{i} \otimes\left(q_{x} s_{1}+q_{y} s_{2}\right) \\
& +q_{x} q_{y}\left(a_{3} \mu_{3}+a_{1} \mu_{1}\right)+g_{23} \mu_{2} s_{3},
\end{aligned}
$$

where all $g$ 's and $a^{\prime} s$ are real parameters.

The $z$-oriented Wilson loop ${ }^{50,51}, \bar{z}_{n}^{ \pm}\left(k_{x}, k_{y}\right)=\left\langle W_{n 0}^{ \pm}|\hat{z}| W_{n 0}^{ \pm}\right\rangle$, is calculated on the (001) surface, where $\left|W_{n 0}^{ \pm}\right\rangle$is the $n$-th Wannier orbit with glide $\mathcal{G}_{x}\left(\mathcal{G}_{y}\right)$ eigenvalues $\pm \mathrm{e}^{-i k_{y}}\left( \pm \mathrm{e}^{-\mathrm{i} k_{x}}\right)$ in the home unit cell, $R=0$ and $\hat{z}$ is the position operator. Figure 2e shows $\bar{z}^{ \pm}$ along the high symmetry $k$ directions for occupied states hosting positive (+sector, red) and negative (-sector, blue) surface glide eigenvalues. Employing the analysis for the two $\mathbb{Z}_{4}$ indexes $\left(X_{x}, X_{y}\right)$ derived in ref. ${ }^{42}$, the bulk topology of our system is $\left(x_{x}, X_{y}\right)=(2,2)$.

\section{Higher-order topological insulator protected by a glide symmetry}

In addition to the topological surface states belonging to the $(d-$ 1) bulk-edge correspondence, i.e., the fourfold Dirac fermion and the $\mathcal{M}_{z}$-protected surface states, the $\mathcal{G}_{x}, \mathcal{G}_{y}$ and $\mathcal{M}_{z}$ symmetries can give rise to higher order $(d-2)$ bulk-edge correspondence.

We have considered the nanorod geometry, shown in Fig. 3a, with open boundary conditions along the [011] and [011] directions, respectively, and periodic boundary conditions along [100]. We find that the nanorod can support two pairs of hinges modes along the intersection lines between the (011) and (011) surfaces and the (011) and (011) surfaces, respectively. None of the above surfaces hosts gapless surface states, since there is no $\mathrm{TCl}$ phase supporting them. As discussed in ref. ${ }^{22}$, the hinge modes formed by the intersection of the (011) and (011) facets are generated via bending the (001) surface along the [001] direction, which, however, preserves the $\mathcal{G}_{y}$ symmetry for the hinges and the entire crystal. The original pair of Dirac cones forming the fourfold Dirac fermion on the (001) surface become gapped with opposite mass terms and reside on the (011) and (011) surfaces, respectively (red and black massive Dirac cones in Fig. 3a). Hence, the surface insulating phases that reside on the two facets differ by an odd $\mathbb{Z}_{2}$ index, leading to the emergence of an odd number of helical hinge modes on the domain wall between the two facets. Similarly, the hinge modes along the intersection of the (011) and (01) facets are generated via bending the (010) surface along the [001] direction, which preserve the $\mathcal{M}_{z}$ symmetry.

Figure $3 \mathrm{~b}$ shows the band structure of the nanorod along the $k_{x}$ symmetry direction where the two pair of hinge modes are denoted in red and cyan, respectively. The distribution in real space of the two types of hinge modes along the [100] direction are displayed in Fig. $3 c$ with the corresponding colors. For the purpose of clarity, onsite potentials $V=0.2 \mathrm{eV}$ are added on the hinges formed by the $\{(011),(0 \overline{1} 1)\}$ and $\{(0 \overline{11}),(01 \overline{1})\}$ facets to better differentiate the two hinge states in energy. The constraint imposed by the glide symmetry $\mathcal{G}_{y}$ allows two possible topologies of hinge bands ${ }^{52}$, the hourglass connectivity and the analog of the quantum spin Hall (QSH) effect (see Fig. 3d). From the hinge modes in cyan displayed in Fig. 3c, we find that the hinge band connectivity is the analog of the quantum spin Hall effect (bottom panel of Fig. 3d).

\section{DISCUSSION}

In ref. ${ }^{37}$, we demonstrated that 9 (Pbam (No. 55), Pccn (No. 56), Pnnm (No. 58), Pnma (No.62), P4/mbm (No. 127), P4/mcn (No.128), $\mathrm{P}_{2} / \mathrm{mbc}$ (No. 135), $P 4_{2} / \mathrm{mnm}$ (No.136), $P 4_{2} / \mathrm{ncm}$ (No. 138)) out of 230 SGs can host CICEs centered at certain TRIM points in the absence of SOC. However, only two wallpaper groups, namely pgg ((001) surface of Pbam (No. 55)) and $p 4 g$ ((001) surface of $P 4 / \mathrm{mbm}$ (No. 127) and $P 4_{2} / m b c$ (No. 135)), containing double-glide lines, can support fourfold Dirac fermions when SOC is introduced. Consequently, only 3 (Pbam (No. 55), P4/mbm (No. 127), and $\mathrm{P}_{2} /$ $m b c$ (No. 135)) of the 9 SGs can support the fourfold Dirac fermions on their (001) surfaces. In SG Pnma (No.55), the CICE resides on the $(010)$ rather than the $(001)$ plane, and the $(010)$ surface $(p g)$ contains a single glide line. Therefore, the (010) TSSs of the corresponding $\mathrm{TCl}$ are hourglass fermions instead. For the rest $5 \mathrm{SGs}$, due to the lack of glide symmetry preserved on their (001) surfaces, the nonsymmorphic fourfold Dirac or hourglass fermions would not be expected.

In summary, the inclusion of SOC in the model Hamiltonian describing our recently proposed family of butterfly-like CICE NLs in SG Pbam (No. 55) ${ }^{37}$ unveils intrinsic connection of the CICE NLSM and the $\mathrm{TCl}$ protected by two glide symmetries. The SOC drives the TSM to a $\mathbb{Z}_{4}=2 \mathrm{TCl}$ with higher order topology, supporting in turn a fourfold Dirac fermion on the (001) surface protected by two coexisting glide symmetries of WG pgg. As a candidate material of this type of $\mathrm{TCl}, \mathrm{Sr}_{2} \mathrm{~Pb}_{3}$ (SG P4/mbm No. 127) has been studied $\mathrm{in}^{42}$. Moreover, its higher order topology is corroborated by the emergence of 1D hinge states protected by glide symmetry, which has never been found before. This intriguing $\mathrm{TCl}$ phase provides a platform for exploring exotic physics, such as the electron transport and thermoelectric effect on the surfaces/hinges. Our proposed glide-protected HOTI may have important implications on the emergence of Majorana zero modes via proximity of the HOTI to a superconductor ${ }^{53}$, which exhibit distinct features compared to those in $\mathrm{Tl} / \mathrm{SC}$ heterostructures. Finally, our EBRs analysis of the tight binding model in Supplementary Discussion demonstrates a rich phase diagram featuring $\mathrm{TCl}$, strong $\mathrm{Tl}$, and obstructed atomic insulator (OAI) phases.

As described in detail in the Supplementary Note, we have carried out systematic ab initio electronic structure calculations to identify material candidates which exhibit (i) a single CICE in the absence of SOC and (ii) fourfold-Dirac TCI/HOTI phase in the presence of SOC. Unfortunately, this task has proved immensely challenging and the calculations failed in finding such an ideal 
$\mathbf{a}$

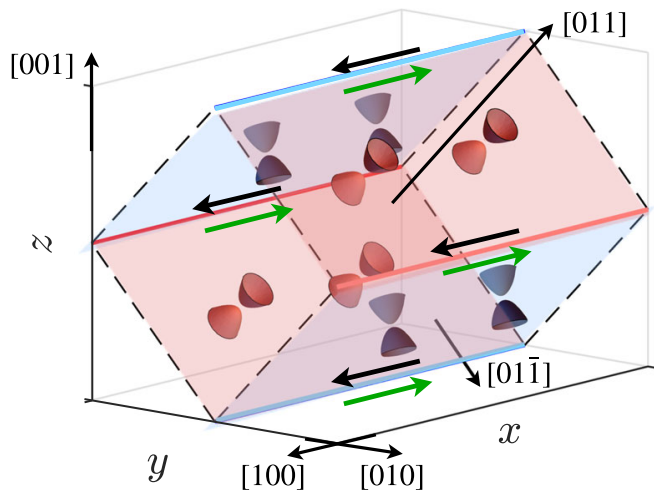

c

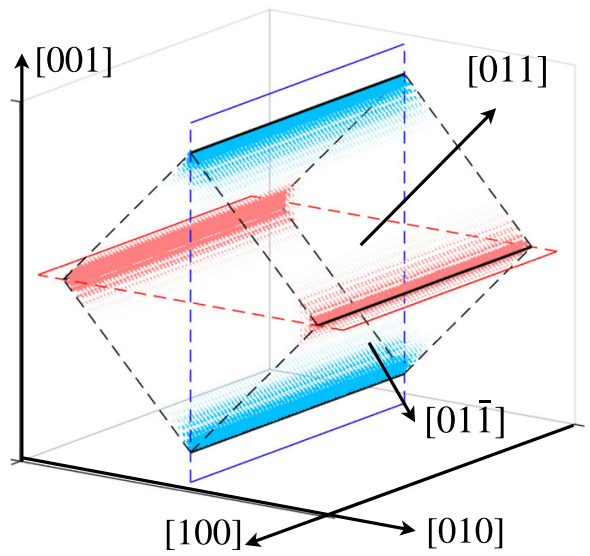

b

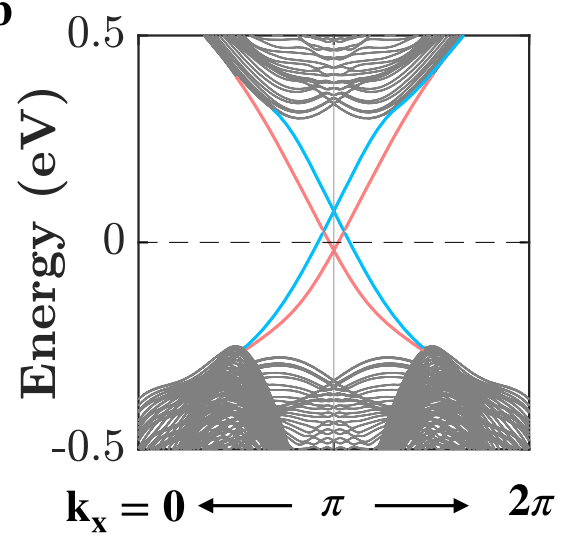

d

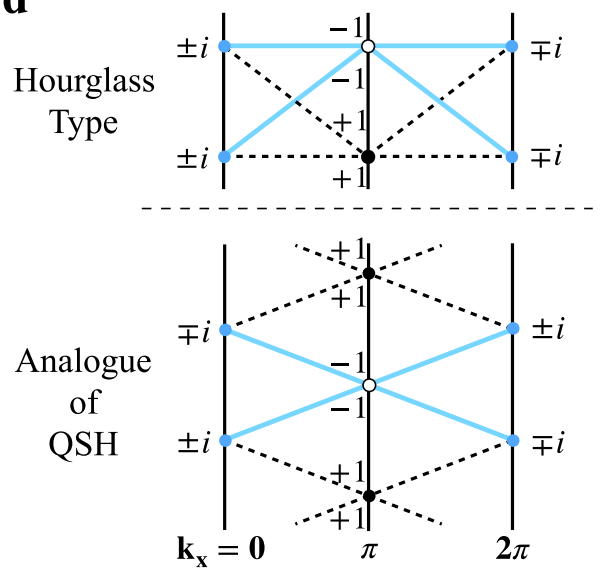

Fig. 3 Bulk-surface-hinge correspondence of the HOTI, and the band connectivity the 1D hinge states. a Geometry of the nanorod, which is periodic along the [100] direction, and finite-size along the [011] and [011] directions. Two pairs of hinge modes (denoted in red and blue) emerge along the intersection lines between the $(011)$ and $(0 \overline{1} 1)$ facets and the (011) and (01) facets, respectively. The first (second) pair of hinge states, denoted by cyan (red), preserves the glide $\mathcal{G}_{y}$ (mirror $\mathcal{M}_{z}$ ) symmetry. b Energy dispersion along $k_{x}$ for the nanorod where the topological hinge modes are denoted with the corresponding cyan and red colors, respectively. $c$ Distribution in real space of the hinge states of the nanorod. For purpose of clarity, on-site potentials $V=0.2 \mathrm{eV}$ are added for the hinges. d Schematic of two symmetry-allowed band connectivities of the hinge states (cyan) protected by glide symmetry $\mathcal{G}_{y}$.

material. This is due to the fact that the band structures in real materials are more complex involving multiple orbitals and bands. Nevertheless, these calculations raise the intriguing question of realizing these quantum states by designing new materials according to our simple model, so that the topological nature can be captured and not buried in the complex band structure. As stated above, we have constructed a minimal (8-band in the presence of SOC) tight-binding model with a bipartite lattice in space group Pbam (No. 55), based on the Wyckoff position 2a with site-symmetry group $2 / m$, and with two orbital per sublattice. Even though we employed the $\left(p_{z}, d_{x y}\right)$-derived orbitals, this choice is not unique and one can equally well use the $\left(p_{z}, s\right)$ derived orbitals. The conditions for the emergence of CICE nodal lines in the absence of SOC are given by Equations (4) and (5) of ref. ${ }^{37}$ with the additional proviso that no other band inversions occur. The conditions to avoid additional band inversions in the presence of SOC are given in the paragraph below Eq. (2) of this work. The artificial system can be designed as layered structure, where these conditions can be satisfied by tuning the interlayer hopping or SOC parameters, such as $\gamma_{1} \lambda_{10,13}$, and $\zeta_{233}$.

\section{DATA AVAILABILITY}

The data that support the findings of this study are available from the corresponding author upon reasonable request.

\section{CODE AVAILABILITY}

The codes are available from the corresponding author upon reasonable request.

Received: 7 August 2020; Accepted: 18 November 2021;

Published online: 13 December 2021

\section{REFERENCES}

1. Kane, C. L. \& Mele, E. J. $Z_{2}$ topological order and the quantum spin Hall effect. Phys. Rev. Lett. 95, 146802 (2005).

2. Kane, C. L. \& Mele, E. J. Quantum spin Hall effect in graphene. Phys. Rev. Lett. 95, 226801 (2005).

3. Bernevig, B. A. \& Zhang, S.-C. Quantum spin Hall effect. Phys. Rev. Lett. 96, 106802 (2006).

4. Fu, L., Kane, C. L. \& Mele, E. J. Topological insulators in three dimensions. Phys. Rev. Lett. 98, 106803 (2007).

5. Moore, J. E. \& L. Balents, L. Topological invariants of time-reversal-invariant band structures. Phys. Rev. B 75, 121306(R) (2007).

6. Roy, R. Topological phases and the quantum spin Hall effect in three dimensions. Phys. Rev. B 79, 195322 (2009).

7. Altland, A. \& Zirnbauer, M. R. Nonstandard symmetry classes in mesoscopic normal-superconducting hybrid structures. Phys. Rev. B 55, 1142 (1997).

8. Kitaev, A. Periodic table for topological insulators and superconductors. AIP Conf. Proc. 1134, 22 (2009)

9. Ryu, S., Schnyder, A. A. P., Furusaki, A. \& Ludwig, A. Topological insulators and superconductors: tenfold way and dimensional hierarchy. New J. Phys. 12, 065010 (2010). 
10. Fu, L. Topological crystalline insulators. Phys. Rev. Lett. 106, 106802 (2011).

11. Slager, R.-J., Mesaros, A., Juričić, V. \& Zaanen, J. The space group classification of topological band-insulators. Nat. Phys. 9, 98-102 (2013).

12. Shiozaki, K. \& Sato, M. Topology of crystalline insulators and superconductors. Phys. Rev. B 90, 165114 (2014).

13. Hsieh, T. H. et al. Topological crystalline insulators in the SnTe material class. Nat. Commun. 3, 982 (2012).

14. Song, Z., Fang, Z. \& Fang, C. (d - 2)-dimensional edge states of rotation symmetry protected topological states. Phys. Rev. Lett. 119, 246402 (2017).

15. Fang, C. \& Fu, L. New classes of topological crystalline insulators having surface rotation anomaly. Sci. Adv. 5, eaat2374 (2019).

16. Zhou, X. et al. Topological crystalline insulator states in the $\mathrm{Ca}_{2} \mathrm{As}$ family. Phys. Rev. B 98, 241104 (2018).

17. Senthil, T. Symmetry-protected topological phases of quantum matter. Annu. Rev. Condens. Matter Phys. 6, 299-324 (2015).

18. Jackiw, R. \& Rebbi, C. Solitons with fermion number. Phys. Rev. D. 13, 3398-3409 (1976).

19. Qi, X.-L. \& Zhang, S.-C. Topological insulators and superconductors. Rev. Mod. Phys. 83, 1057-1110 (2011).

20. Benalcazar, W. A., Bernevig, B. A. \& Hughes, T. L. Quantized electric multipole insulators. Science 357, 61-66 (2017).

21. Benalcazar, W. A., Bernevig, B. A. \& Hughes, T. L. Electric multipole moments, topological multipole moment pumping, and chiral hinge states in crystalline insulators. Phys. Rev. B 96, 245115 (2017).

22. Schindler, F. et al. Higher-order topological insulators. Sci. Adv. 4, eaat0346 (2018).

23. Schindler, F. et al. Higher-order topology in bismuth. Nat. Phys. 14, 918-924 (2018).

24. Wang, Z., Wieder, B. J., Li, J., Yan, B. \& Bernevig, B. A. Higher-order topology, monopole nodal lines, and the origin of large Fermi arcs in transition metal dichalcogenides $x \mathrm{te}_{2}(x=$ Mo, W). Phys. Rev. Lett. 123, 186401 (2019).

25. Park, M. J., Kim, Y., Cho, G. Y. \& Lee, S. Higher-order topological insulator in twisted bilayer graphene. Phys. Rev. Lett. 123, 216803 (2019).

26. Zhang, R.-X., Wu, F. \& Das Sarma, S. Möbius insulator and higher-order topology in $\mathrm{mnbi}_{2 n} \mathrm{te}_{3 n+1}$. Phys. Rev. Lett. 124, 136407 (2020).

27. Ren, Y., Qiao, Z. \& Niu, Q. Engineering corner states from two-dimensional topological insulators. Phys. Rev. Lett. 124, 166804 (2020).

28. Burkov, A. A., Hook, M. D. \& Balents, L. Topological nodal semimetals. Phys. Rev. B 84, 235126 (2011)

29. Fang, C., Weng, H., Dai, X. \& Fang, Z. Topological nodal line semimetals. Chin. Phys. B 25, 117106 (2016).

30. Bzdušek, T., Wu, Q. S., Rüegg, A., Sigrist, M. \& Soluyanov, A. A. Nodal-chain metals. Nature 538, 75-78 (2016).

31. Chang, G. et al. Topological hopf and chain link semimetal states and their application to $\mathrm{CO}_{2} \mathrm{MnGa}$. Phys. Rev. Lett. 119, 156401 (2017).

32. Yan, Z. et al. Nodal-link semimetals. Phys. Rev. B 96, 041103 (2017).

33. Bi, R., Yan, Z., Lu, L. \& Wang, Z. Nodal-knot semimetals. Phys. Rev. B 96, 201305 (2017).

34. Kim, Y., Wieder, B. J., Kane, C. L. \& Rappe, A. M. Dirac line nodes in inversionsymmetric crystals. Phys. Rev. Lett. 115, 036806 (2015).

35. Huang, H., Liu, J., Vanderbilt, D. \& Duan, W. D. Topological nodal-line semimetals in alkaline-earth stannides, germanides, and silicides. Phys. Rev. B 93, 201114(R) (2016).

36. Xu, Q., Yu, R., Fang, Z., Dai, X. \& Weng, H. Topological nodal line semimetals in the $\mathrm{CaP}_{3}$ family of materials. Phys. Rev. B 95, 045136 (2017).

37. Zhou, X. et al. Novel family of topological semimetals with butterflylike nodal lines. Phys. Rev. B 104, 125135 (2021).

38. Po, H. C., Vishwanath, A. \& Watanabe, H. Symmetry-based indicators of band topology in the 230 space groups. Nat. Commun. 8, 50 (2017).

39. Kruthoff, J., de Boer, J., van Wezel, J., Kane, C. L. \& Slager, R.-J. Topological classification of crystalline insulators through band structure combinatorics. Phys. Rev. X 7, 041069 (2017).

40. Khalaf, E., Po, H. C., Vishwanath, A. \& Watanabe, H. Symmetry indicators and anomalous surface states of topological crystalline insulators. Phys. Rev. $\times \mathbf{8}$, 031070 (2018).

41. Song, Z., Zhang, T., Fang, Z. \& Fang, C. Quantitative mappings between symmetry and topology in solids. Nat. Commun. 9, 3530 (2018).

42. Wieder, B. J. et al. Wallpaper fermions and the nonsymmorphic Dirac insulator. Science 361, 246-251 (2018).

43. Young, S. M. \& Kane, C. L. Dirac semimetals in two dimensions. Phys. Rev. Lett. 115, 126803 (2015).

44. Bradlyn, B. et al. Topological quantum chemistry. Nature 547, 298-305 (2017).

45. Vergniory, M. G. et al. Graph theory data for topological quantum chemistry. Phys. Rev. E 96, 023310 (2017)
46. Elcoro, L. et al. Double crystallographic groups and their representations on the Bilbao Crystallographic Server. J. Appl. Crystallogr. 50, 1457 (2017).

47. Aroyo, M. I. et al. Bilbao Crystallographic Server: I. Databases and crystallographic computing programs. Z. f.ür. Kristallographie-Crystalline Mater. 221, 15-20 (2006).

48. Aroyo, M. I., Kirov, A., Capillas, C., Perez-Mato, J. M. \& Wondratschek, H. Bilbao Crystallographic Server. II. Representations of crystallographic point groups and space groups. Acta Crystallogr. Sect. A 62, 115-128 (2006).

49. Aroyo, M. I. et al. Crystallography online: Bilbao crystallographic server. Bulgarian Chem. Commun. 43, 183 (2011).

50. Yu, R., Qi, X. L., Bernevig, A., Fang, Z. \& Dai, X. Equivalent expression of $\mathbb{Z}_{2}$ topological invariant for band insulators using the non-abelian berry connection. Phys. Rev. B 84, 075119 (2011).

51. Taherinejad, M., Garrity, K. F. \& Vanderbilt, D. Wannier center sheets in topological insulators. Phys. Rev. B 89, 115102 (2014)

52. Wang, Z., Alexandradinata, A., Cava, R. J. \& Bernevig, B. A. Hourglass fermions. Nature 532, 189-194 (2016).

53. Hsu, C.-H., Stano, P., Klinovaja, J. \& Loss, D. Majorana Kramers pairs in higher-order topological insulators. Phys. Rev. Lett. 121, 196801 (2018).

\section{ACKNOWLEDGEMENTS}

The work at CSUN was supported by NSF-Partnership in Research and Education in Materials (PREM) Grant No. DMR-1828019. H.L. acknowledges the support by the Ministry of Science and Technology (MOST) in Taiwan under grant number MOST 109-2112-M-001-014-MY3. The work of J.L.M. has been supported by Spanish Science Ministry grant PGC2018-094626-B-C21 (MCIU/AEI/FEDER, EU) and Basque Government grant IT979-16. M.G.V. thanks support from DFG INCIEN2019-000356 from Gipuzkoako Foru Aldundia. M.G.V. and M.I. acknowledges the Spanish Ministerio de Ciencia e Innovacion (grant number PID2019-109905GB-C21).

\section{AUTHOR CONTRIBUTIONS}

X.Z. designed research. X.Z., C.-H.H., M.I., C.-Y.H., J.M.L., M.G.V., H.L. and N.K. performed research. X.Z., C.-H.H., M.I., C.-Y.H., and J.M.L. analyzed data. X.Z. and C.-H.H. drafted the main text, whereas J.M.L, M.I. and X.Z. drafted the supplementary information. All authors contributed to the editing of the manuscript.

\section{COMPETING INTERESTS}

The authors declare no competing interests.

\section{ADDITIONAL INFORMATION}

Supplementary information The online version contains supplementary material available at https://doi.org/10.1038/s41524-021-00672-9.

Correspondence and requests for materials should be addressed to Xiaoting Zhou or Nicholas Kioussis.

Reprints and permission information is available at http://www.nature.com/ reprints

Publisher's note Springer Nature remains neutral with regard to jurisdictional claims in published maps and institutional affiliations.

Open Access This article is licensed under a Creative Commons Attribution 4.0 International License, which permits use, sharing, adaptation, distribution and reproduction in any medium or format, as long as you give appropriate credit to the original author(s) and the source, provide a link to the Creative Commons license, and indicate if changes were made. The images or other third party material in this article are included in the article's Creative Commons license, unless indicated otherwise in a credit line to the material. If material is not included in the article's Creative Commons license and your intended use is not permitted by statutory regulation or exceeds the permitted use, you will need to obtain permission directly from the copyright holder. To view a copy of this license, visit http://creativecommons. org/licenses/by/4.0/

(c) The Author(s) 2021 